\title{
Diseño de un instrumento para la identificación del matoneo
}

\author{
Juan Carlos Velásquez Díaz \\ Especialista en Psicología Clínica de la Niñez y la Adolescencia \\ Universidad de la Sabana. \\ Chía, Colombia \\ Correo electrónico: juanveldi@unisabana.edu.co
}

Lida Darley Pineda Rodríguez

Especialista en Psicología Clínica de la Niñez y la Adolescencia

Universidad de la Sabana. Chía, Colombia

Correo electrónico: lidapiro@unisabana.edu.co
Recibido: 11/03/2016

Evaluado: $17 / 05 / 2016$

Aceptado: 24/06/2016

Objetivo: diseñar un instrumento que permita identificar matoneo dentro de contextos educativos, teniendo en cuenta los tres actores presentes en esta problemática (intimidador, víctima y observador) y diferentes tipos de agresión (física, social y verbal), lo anterior debido a que Colombia no cuenta con instrumentos de evaluación validados que identifiquen dichos aspectos de manera adecuada. Método: La prueba consta de 35 ítems y fue sometida a evaluación por jueces expertos, para su posterior modificación y aplicación a una muestra de 300 estudiantes, de ambos sexos y con edades entre los 9 y 17 años de un colegio privado del municipio de Subachoque Cundinamarca. Resultados: El instrumento fue evaluado mediante criterios de confiabilidad y validez, obteniendo un adecuado soporte estadístico, logrando establecer para la prueba un índice de confiabilidad de .961 mediante coeficiente Alpha de Cronbach. Conclusión: Los análisis estadísticos realizados a la prueba diseñada indican que esta cumple adecuadamente con los criterios de confiabilidad y validez.

\section{Palabras clave}

Matoneo, evaluación psicológica, validez y confiabilidad.

8 Para citar este artículo: Velásquez, J., \& Pineda, L. (2016). Diseño de un instrumento para la identificación del matoneo. Informes Psicológicos, 16(2), pp. 121-141. http://dx.doi.org/10.18566/infpsicv16n2a08 


\title{
Design of an instrument for the identification of bullying
}

\begin{abstract}
Objective: to design an instrument that allows to identify bullying within educational contexts, taking into account the three main actors present in this issue (bully, victim, and observer) and different types of aggression (physical, social and verbal). Colombia does not have validated assessment instruments to identify these aspects adequately. Method: the test consists of 35 items; it was submitted for evaluation by expert judges, and it was later modified and applied to a sample of 300 students of both sexes, aged 9 to 17, belonging to a private school in the municipality of Subachoque in the Cundinamarca department. Results: the instrument was evaluated using reliability and validity criteria, obtaining a suitable statistical support, with a reliability index of .961 using Cronbach's Alpha coefficient. Conclusion: statistical analyses applied to the test indicate that it adequately meets the criteria of reliability and validity.
\end{abstract}

Keywords bullying, psychological assessment, validity and reliability.

\section{Desenho de um instrumento para a identificação do acosso escolar}

Resumo

Objetivo: desenhar um instrumento que permita identificar o acosso escolar dentro de contextos educativos, tendo em conta os três atores presentes nesta problemática (intimidador, vítima e observador) e diferentes tipos de agressão (física, social e verbal), o anterior devido a que na Colômbia não conta com instrumentos de avaliação validados que identifiquem ditos aspectos de maneira adequada. Método: A prova consta de 35 itens e foi submetida a avaliação por juízes expertos, para sua posterior modificação e aplicação a uma amostra de 300 estudantes, de ambos sexos e com idades entre os 9 e 17 anos de um colégio particular do município de Subachoque Cundinamarca. Resultados: 0 instrumento foi avaliado mediante critérios de confiabilidade e validez, obtendo um adequado suporte estatístico, conseguindo estabelecer para a prova um índice de confiabilidade de .961 mediante coeficiente Alpha de Cronbach. Conclusão: Os análise estatísticos realizados na prova desenhada indicam que esta cumpre adequadamente com os critérios de confiabilidade e validez. 


\section{ntroducción}

El matoneo es uno de los temas de interés que dentro del campo de la psicología clínica y educativa centra mayor atención, no solo en nuestro país sino a nivel mundial, en donde se pretende dar luces para poner freno a una serie de abusos físicos, verbales y psicológicos entre estudiantes de instituciones educativas; este es un tema de gran relevancia en nuestros días, pero que se presenta desde hace muchos años. Se puede decir que en todos los tiempos los niños han sido víctimas de abusos, ya sean físicos, psicológicos, entre otros.

Por tal razón, el Ministerio de Educación Nacional ha desarrollado la ley antimatoneo (ley 1620 del 15 de marzo de 2013) mediante la cual se crea el sistema nacional de convivencia escolar y formación para los derechos humanos, la educación para la sexualidad, la prevención y mitigación de la violencia escolar; que tiene como objetivo promover y fortalecer la formación ciudadana y el ejercicio de los derechos humanos, sexuales y reproductivos de los estudiantes, de los niveles educativos de preescolar, básica y media, previniendo y mitigando la violencia escolar y el embarazo en los adolescentes colombianos. Algunas investigaciones realizadas (Mera, 2014; Rodríguez, 2012) se centran en la identificación de las víctimas y de las consecuencias físicas y psicológicas de las mismas, pero no logran tener un impacto diferente que el dar a conocer datos estadísticos y sociales.

Por lo expuesto anteriormente se identifica como favorable diseñar un instrumento que se adapte a las necesidades y características de la población colombiana, teniendo en cuenta que la gran mayoría de instrumentos que se utilizan en nuestro país evalúan comportamientos de agresión, más no de matoneo; así mismo no incluyen los diferentes actores del evento y/o no incluyen las diferentes formas de agresión, ni la permanencia de este fenómeno en el tiempo (característica del matoneo).

\section{Conceptualización del matoneo}

Uno de los primeros autores en plantear el concepto del matoneo fue Olweus (1973), quien se comenzó a interesar por los casos de violencia escolar presentes en su país (Noruega) y especialmente tras el suicidio de algunos jóvenes en 1982 por motivos que aparentemente tenían relación directa con una serie de abusos dentro del contexto educativo.

Olweus (2006) define el matoneo como las acciones negativas que se producen de forma repetida en el tiempo, y considera que una acción tal se produce cuando un estudiante, de manera intencionada, causa un daño o hace sentir incomodo a alguno de sus pares. Dicha agresión puede ser obra de un solo individuo o de un grupo de personas. No obstante, en cualquier caso, para poder hablar concretamente de matoneo debe evidenciarse un desequilibrio de fuerzas de relación de poder asimétrica; es decir, que la persona que es sometida a este tipo de violencia presenta dificultad para defenderse, por lo que se podría decir que se encuentra vulnerable a la intimidación. Para que se considere como 
matoneo, en definitiva se requiere que la situación cumpla con cuatro condiciones: los episodios de hostigamiento deben darse entre compañeros del colegio, ha de presentarse en un marco de desequilibrio del poder, es necesario que sea un fenómeno reiterativo y finalmente, tiene que ser intimidatorio (Olweus, 2006).

Cerezo (2006) menciona que el matoneo no se trata de un episodio esporádi$\mathrm{co}$, sino persistente, que puede durar semanas, meses e incluso años. El matoneo "suele comenzar con chistes e insultos, donde se observa que la víctima se va aislando del resto de los estudiantes, estas acciones adquieren poco a poco más violencia" (Velásquez, Pineda, \& Castro, 2013, p. 15). El patio, los pasillos, y los baños de los colegios son los sitios o espacios considerados por los expertos como los puntos principales donde se presenta matoneo, ya que estos en ocasiones suelen ser los menos vigilados y controlados por los profesores (Ricou, 2005). Autores como Chaves, Morales y Ortega (2015) identificaron en un estudio realizado que los lugares en los cuales existe mayor prevalencia de matoneo son: las aulas de clase, los baños y los pasillos; aunque resaltan que este fenómeno también se presenta en lugares cercanos al colegio.

Se dice que millones de niños sufren de matoneo en sus colegios, actualmente es un problema común alrededor del mundo. En países como Inglaterra y Estados Unidos existen diversas organizaciones y campañas que buscan ayudar a niños víctimas de matoneo; en España la primera condena por esta problemática se dio en el año 2004, cuando un joven de 14 años se suicidó por recibir maltrato constante en el contexto educativo, distintos casos como este han generado investigaciones para identificar la gravedad del tema y para que se tomen acciones preventivas (Jaramillo, 2012).

Garaigordobil (2011) menciona que en la revisión de los estudios realizados sobre matoneo se observa que las características y formas de este fenómeno no presentan una amplia variación de un país a otro; por lo general, todos los estudios evidencian la existencia del matoneo entre iguales, por lo que se puede concluir que es una realidad que está presente en todos los colegios del mundo, así mismo este autor expone que en la revisión realizada se logra constatar la incidencia de la violencia escolar sin que existan diferencias notables por el contexto geográfico, cultural o educativo; también resalta que en la revisión de otros estudios (Cerezo, 2009; Del Rey \& Ortega, 2008; Garaigordobil \& Oñederra, 2008, 2010; Gutiérrez, Barrios, De Dios, Montero \& Del Barrio, 2008; Pérez \& Gázquez, 2010) se permite observar un porcentaje promedio de victimización grave entre el 3\% y el 10\%, y porcentajes de estudiantes que sufren conductas violentas que oscilan entre un $20 \%$ y un $30 \%$; finalmente concluye que la mayoría de los implicados son varones, el tipo de acoso más frecuente es el verbal y el lugar del colegio donde más agresiones se producen es el patio.

Luna (2006) indica que el maltrato y la violencia entre estudiantes se está convirtiendo en un fenómeno preocupante en las escuelas de América Latina, según expertos de seis países reunidos el 25 de octubre de 2007 en un panel en Bogotá en donde se divulgó una encuesta sobre el tema se describieron los siguientes resultados: un $22.9 \%$ de los estudiantes indicó haber sido agredido verbalmente por sus compañeros, el 15.6 \% fue objeto 
de robos o daños en sus pertenencias, un $9.6 \%$ recibió golpes y un $8.2 \%$ fue amenazado. La encuesta fue realizada en Argentina, México, Brasil, Chile, Puerto Rico y Colombia (Velásquez, Pineda, \& Castro, 2013).

En Colombia, específicamente la Alcaldía de Bogotá, el Departamento Nacional de Estadística (DANE), con la participación Investigadores de la Universidad de los Andes realizaron una encuesta entre marzo y abril de 2006 en 807 instituciones educativas públicas y privadas de los diferentes estratos socioeconómicos, con el fin de obtener datos relacionados con la violencia escolar en la capital del país-, esta fue aplicada a 826.455 estudiantes. Este estudio señala que 13.089 estudiantes fueron testigos de ataques con algún tipo de arma, por lo menos 5.191 fueron intimidados, un 33\% de los encuestados fueron agredidos físicamente por sus compañeros, y un 5\% fueron remitidos a centros médicos para recibir atención urgente por agresiones.

\section{Clases, actores y consecuencias del matoneo}

Luna (2006) describe las formas más comunes en las cuales se presenta el fenómeno del matoneo. El primero es maltrato directo, que incluye daño físico como patadas, quemaduras, puñetazos, empujones, pellizcos, zancadillas, entre otros, o verbal: como usar apodos, realizar insultos, amenazas y chantajes. El maltrato indirecto, esconder útiles, dañar objetos de la víctima, robarle, arruinar los trabajos, entre otros; o de carácter verbal, como expandir rumores para desprestigiar a la víctima, ignorarla o excluirla socialmente. Finalmente se encuentra el cyberbulliyng, en donde por lo general se envían mensajes intimidatorios, que se utilizan para humillar y denigrar a la víctima a través de fotos digitales, videos con burlas y falsos rumores.

Dentro de esta problemática se encuentran inmersos tres actores (víctimas, intimidadores y observadores), por lo que se describirán las particularidades de cada uno de ellos. Las características de las víctimas de matoneo son expuestas por Cerezo (2006) y Kumpulainen (2008) quienes destacan que la edad de éstas es menor a la de los intimidadores, en cuanto a su apariencia física suelen presentar complexión débil y/o obesidad, el rendimiento académico es superior a su grupo de pares, las relaciones familiares son algo mejores que las que mantienen los intimidadores. Usualmente se percibe en ellos una baja autoestima, actitudes pasivas, problemas psicosomáticos, depresión, ansiedad y en muchos de ellos pensamientos suicidas.

Voors (2005, citado por Velásquez, Pineda, \& Castro, 2013) describe las características y comportamientos más comunes entre los niños y adolescentes que ejercen matoneo, destacando que: tienen gusto por hablar despectivamente de otras personas, no les importa lastimar los sentimientos ajenos, presentan frecuentemente desacato de la autoridad, manifiestan falta de respeto por el sexo opuesto, realizan bromas o chistes sobre violaciones y otras agresiones contra las mujeres, les gusta pelear, no toleran las equivocaciones, mienten para evitar responsabilidades, maltratan intencionalmente mascotas y otros animales, no 
confían fácilmente en otras personas, les gusta mantener una postura de superioridad con respecto a los demás niños y se enojan con facilidad.

Autores como Rodríguez, Seoane y Pedreira (2008) plantean que los observadores evitan intervenir en situaciones de matoneo para protegerse de los intimidadores, convirtiéndose de esta forma en cómplices del abuso. Avilés (2009, citado por Erazo, 2012) indica que generalmente el observador está presente de forma pasiva, es decir, que no genera ningún tipo de intervención o mediación en el acto de matoneo, algunos de ellos se muestran poco sensibles ante los hechos o no experimentan empatía por las víctimas.

De acuerdo con Arellano (2008), el observador juega en ocasiones el papel de cómplice, ya que continuamente aprueba y acompaña con su presencia el acto de matoneo. Este tipo de espectador es muy cercano al agresor y puede terminar emulándolo en busca de recompensa social.

Sin duda, las consecuencias del matoneo son uno de los aspectos a los cuales más se debe prestar atención. Arroyave (2012) indica que muchas de las consecuencias del matoneo van mucho más allá de la sensación de incomodidad que se puede vivir entre los estudiantes y en los mismos colegios, donde las secuelas se pueden observar años después, tanto en el estrés psicológico general, como en trastornos psiquiátricos específicos en la edad adulta. Entre las consecuencias más impactantes del matoneo está la ideación e intento de suicidio, que se presenta tanto en víctimas como en intimidadores.
En general las investigaciones sobre los efectos del matoneo demuestran lo perjudicial y destructivo que es, ya que las víctimas tienden a presentar baja autoestima, depresión, ansiedad, algunos suelen ser cautelosos y reservados, normalmente estos estudiantes se muestran encerrados en sí mismos, preocupados y temerosos ante situaciones nuevas, mostrando una introversión marcada, son menos felices en el colegio, más solitarios y tienen menos amigos; como consecuencia tienen más posibilidades de abandonar sus estudios, especialmente las niñas se ven afectadas por el hecho de ser evitadas socialmente o de ser evaluadas negativamente por parte de sus iguales (Olweus, 2000).

\section{Diseño de pruebas psicológicas}

La prueba diseñada consta de 35 ítems, los cuales permiten identificar los tres actores del matoneo y las diferentes formas de agresión. Se hace relevante indicar que la prueba fue sometida a evaluación por criterios de confiabilidad y validez, con el fin de brindar un soporte estadístico y metodológico a los profesionales que deseen replicarla en alguna muestra específica.

Teniendo en cuenta que el objetivo principal de la investigación es el diseño de un instrumento que permita identificar los actores del matoneo, se inicia este apartado definiendo que es una prueba. Sattler (2003, citado por Bonilla, Osorio, \& Triana, 2010) indican que la palabra prueba se aplica a cualquier procedimiento estándar que se utiliza para medir la manifestación de una conducta, y en tal 
sentido por "procedimiento estándar" se entienden los siguientes preceptos: contenido reactivo (elegido para representar el campo de interés), procedimiento de aplicación (instrucciones, redacción de los reactivos, preguntas de sondeo, registro de respuestas y límites de tiempo), criterios de calificación (pautas objetivas que se proporcionan para evaluar las respuestas).

Bonilla et al. (2010) describen que la medición psicológica mide lo intangible, aunque nunca es tan precisa como la física, transmite información significativa e importante sobre la gente y está diseñada para medir atributos de la persona (inteligencia, habilidades motrices, capacidad de lectura, comportamiento de adaptación, extraversión o introversión, entre otros) y genera una puntuación que refleja el atributo. Uno de los principales objetivos de la psicometría es estudiar la confiabilidad y la validez de las pruebas psicológicas, por medio de criterios que permiten establecer la solidez de las mismas; por esta razón se hace pertinente describir los aspectos referentes a la confiabilidad y validez.

Kerlinger y Lee (2002) mencionan que la confiabilidad se refiere a la consistencia o estabilidad de una medida. Hernández, Fernández y Baptista (2006) indican que la confiabilidad de un instrumento de medición está relacionada con el grado en que su aplicación repetida a un mismo sujeto produce resultados iguales, es decir, que si en su aplicación a una misma persona en repetidas ocasiones genera resultados distintos no podría decirse de que es confiable, debido a que sus resultados no serían consistentes. De acuerdo con Aiken (1996) la confiabilidad o consistencia de un de un test es la precisión o exactitud con que el test mide lo que debe medir en una población determinada y en las condiciones normales de aplicación.

Existen diversas formas para calcular la confiabilidad de un instrumento, todos estos métodos utilizan fórmulas que generan coeficientes que oscilan entre $0 \mathrm{y}$ 1 , en donde un coeficiente de 0 significa nula confiabilidad y 1 significa o representa un máximo de confiabilidad; puntajes entre .70 y .80 reflejan niveles aceptables. Los procedimientos que más se utilizan para determinar la confiabilidad mediante un coeficiente son: a) Medida de estabilidad o test-retest. b) Método de formas alternativas o paralelas. c) Método de mitades partidas. d) Coeficiente alfa de Cronbach e) Coeficiente KR20 (Hernández et al., 2006).

Es importante describir el Coeficiente Alpha de Cronbach, debido a que éste es el procedimiento utilizado para establecer la confiabilidad de la prueba que se diseñó. Cohen y Swerdlik (2001) mencionan que el coeficiente Alpha, fue propuesto por Lee J. Cronbach en el año 1951. Este coeficiente representa las fórmulas KR-20 y KR-21, desarrolladas en 1937 por Kuder y Richardson (Kerlinger \& Lee, 2002), que sólo se podían aplicar o pruebas de respuesta dicotómica. Con la creación del Alpha de Cronbach, los investigadores fueron capaces de evaluar la confiabilidad o consistencia interna de un instrumento constituido por una escala Likert, o cualquier escala de opciones múltiples, desde ese momento se utiliza dicho coeficiente en la evaluación de la estructura interna de los test (Cronbach, 1951).

Uno de los aspectos que genera en ocasiones mayor duda en los 
investigadores se refiere a cuál debe ser el valor mínimo del coeficiente Alpha de Cronbach. Arbigay (2006) describe en sus estudios que no hay un criterio uniforme, se podría decir que un parámetro aceptable es un mínimo de .70. Posiblemente, lo más recomendable al interpretar el coeficiente de confiabilidad sea considerar que tan próximo está al valor máximo (1), evaluar el margen de error y considerar las distintas circunstancias que involucren el uso del instrumento. Para Kerlinger y Lee (2002) la mayoría de investigadores consideran como regla general que los índices que confiabilidad no deben ser inferiores a .80 .

Como se mencionaba anteriormente, el segundo aspecto o criterio que debe tener un test se refiere a la validez. Hernández et al. (2006) la definen como el grado en que un instrumento mide la variable que pretende medir. Diferentes autores realizan clasificaciones de la validez, en donde por lo general utilizan tres categorías: de contenido, de criterio y de constructo.

a). Validez de contenido: Autores como Arbigay (2006) y Aroca (1999, citado por Delgado et al., 2002) indican que esta validez está representada por el grado en que una prueba representa el universo de estudio. Por lo que deben seleccionarse los indicadores e ítems de tal manera que estos respondan a las características particulares de la evaluación. El método que más se utiliza para estimar la validez de contenido es el denominado "Juicio de Expertos", el cual consiste en seleccionar un número impar (3 ó 5) de jueces, quienes tienen la labor de leer y evaluar cada uno de los ítems del instrumento, de acuerdo a criterios de redacción, relevancia y pertinencia.

b). Validez de criterio: es una medida del grado en que una prueba está relacionada con otro criterio de evaluación, del mismo constructo o conducta. Es de suponer que el criterio con el que se está comparando la prueba tiene un valor intrínseco como medida de algún rasgo o característica (Delgado et al., 2002).

c). Validez de constructo: determina a través del procedimiento de análisis de factores en qué medida los resultados de una prueba se relacionan con la definición de constructos o atributos para explicar un fenómeno. Para el presente estudio se tiene como referencia la definición dada por Olweus (2006) sobre el matoneo. El método más utilizado para establecer la validez de constructo es el análisis factorial, esta técnica analiza las intercorrelaciones de un conjunto de datos, para establecer determinadas agrupaciones de ítems correlacionados entre sí, las cuales remiten a factores subyacentes, que no son observables. El análisis factorial se utiliza no solo para evaluar la validez del instrumento, sino también su construcción, este análisis puede ser tanto exploratorio como confirmatorio, de acuerdo a la necesidad de los investigadores. (Arbigay, 2006).

En la construcción de una prueba se deben tener en cuenta los aspectos descritos por Tornimbeni, Pérez, Olaz y Fernández (2004), en donde se destacan las siguientes recomendaciones: diseñar ítems que tengan coherencia y relación con el objetivo de medición, redactar 
ítems cortos, evitar oraciones ambiguas, así como el uso de términos absolutos (nunca, siempre, etc.) y utilizar un lenguaje acorde al nivel educativo y de desarrollo de la población.

Por las razones expuestas anteriormente, el objetivo general de la investigación fue diseñar un instrumento que permita identificar los tres actores (víctima, intimidador y observador) que se presentan dentro del fenómeno del Matoneo, teniendo en cuenta las diferentes dimensiones de agresión (física, social y verbal), brindando una herramienta que facilite la identificación e intervención en dicha problemática. Para lograr el objetivo propuesto. El instrumento de evaluación diseñado fue sometido a evaluación de criterios de confiabilidad, por medio del Coeficiente Alpha de Cronbach, validez de contenido mediante análisis de jueces expertos y validez de constructo, mediante la utilización del análisis factorial exploratorio y confirmatorio; se establecieron estadísticos descriptivos, índices de correlación entre ítems, la medida de adecuación muestral de Kaiser-MeyerOlkin (KMO), la prueba de esfericidad de Bartlett, la varianza total explicada y la matriz factorial de cada una de las combinaciones entre factores y dimensiones de la agresión.

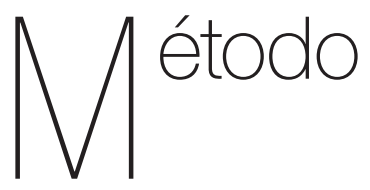

\section{Diseño}

La investigación se realizó bajo un diseño tecnológico psicométrico con análisis de datos descriptivos (Juárez \& Montejo,
2006), buscando que el instrumento de evaluación desarrollado cumpliera con los criterios de confiabilidad y validez.

\section{Participantes}

Una vez sometido el instrumento diseñado a evaluación por jueces expertos, se aplicó a 300 estudiantes, 159 hombres y 141 mujeres, del Colegio Liceo Campestre Thomas de Iriarte del municipio de Subachoque. La muestra seleccionada se encontraba en un rango de edad entre los 9 y 17 años, con nivel académico entre grados 5 y 9. La prueba fue aplicada al total de estudiantes matriculados entre los grados mencionados anteriormente.

\section{Instrumento}

La herramienta Estadístical Package for the Social Sciences (SPSS) 2.0 fue utilizada para establecer el Coeficiente de Confiabilidad Alfa de Cronbach y para realizar en Análisis Factorial de los datos obtenidos en la aplicación del instrumento a la muestra seleccionada.

\section{Procedimiento}

Para el desarrollo de la investigación y construcción del instrumento de evaluación se contó con el apoyo de tres jueces expertos (dos expertos en el tema del matoneo y un experto metodológico), quienes evaluaron la prueba inicialmente diseñada con respecto a las variables de redacción, relevancia y pertinencia de cada uno de los ítems. 
La investigación se realizó en diferentes fases, las cuales son descritas a continuación:

Fase 1. Diseño del instrumento de evaluación, redacción de 15 ítems por cada uno de los actores del matoneo (intimidador, víctima y observador), incluyendo tres dimensiones de agresión (física, verbal y social), con 5 ítems por cada una; para un total de 45 enunciados.

Fase 2. Determinación de la forma de corrección de la prueba.

Fase 3. Realización de la validación de contenido de la prueba, por medio de la evaluación por tres jueces expertos.

Fase 4. Establecimiento del criterio de Confiabilidad con base en el análisis estadístico de los resultados de los jueces expertos (Coeficiente Alfa de Cronbach) y modificación de ítems de acuerdo a las sugerencias de los mismos.

Fase 5. Aplicación del instrumento a la muestra seleccionada de 300 estudiantes del Colegio Liceo Campestre Thomas de Iriarte, previa autorización por parte de las directivas del Colegio.

Fase 6. Identificación de la validez de constructo, mediante la utilización del análisis factorial exploratorio y confirmatorio; se establecieron estadísticos descriptivos, índices de correlación entre ítems, la medida de adecuación muestral de Kaiser-Meyer-Olkin (KMO), la prueba de esfericidad de Bartlett, la varianza total explicada y la matriz factorial de cada una de las combinaciones entre factores y dimensiones de la agresión.
Fase 7. Determinación del criterio de Confiabilidad una vez depurada la prueba, eliminando los ítems que no discriminaban adecuadamente, se realizó nuevamente el análisis mediante el Coeficiente Alpha de Cronbach.

Fase 8. Descripción, organización y análisis de los datos obtenidos al haber sometido la prueba a evaluación mediante criterios de confiabilidad y validez.

Fase 9. Consolidación de la prueba final de acuerdo a los resultados obtenidos.

\section{Consideraciones Eticas}

Teniendo en cuenta que el tipo de investigación realizada utiliza un diseño Tecnológico Psicométrico, para el presente trabajo se tuvieron en cuenta los lineamientos y principios básicos que menciona el Código Deontológico que rige el desarrollo y el ejercicio profesional de la Psicología en Colombia, resaltando los principios de beneficencia, no maleficencia y confidencialidad, por lo que se contó con el apoyo y aprobación de psicólogos expertos para la evaluación de la prueba, la autorización de la rectora del Colegio Liceo Campestre Thomas de Iriarte, la aceptación de los estudiantes para responder la prueba, quienes lo hicieron de manera libre y sin ningún tipo de condición previa, aclarando que los resultados tenían únicamente un fin investigativo. 


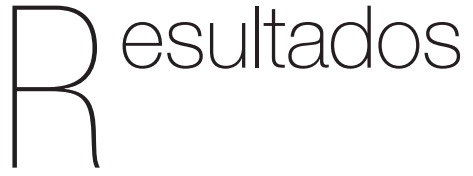

La evaluación realizada por los 3 jueces expertos y posteriormente sometida al análisis estadístico, obtuvo un coeficiente de confiabilidad Alfa de Cronbach de .959 (Ver Tabla 1). De acuerdo con las sugerencias de los jueces, se modificó la redacción de los ítems 4, 21, 25, 30, 39, 40,41 , y 42.

Tabla 1.

Índice de Confiabilidad por evaluación de jueces expertos

\begin{tabular}{|c|c|c|c|c|}
\hline & $\begin{array}{l}\text { Media de la escala si } \\
\text { se elimina el elemento }\end{array}$ & $\begin{array}{l}\text { Varianza de la escala si } \\
\text { se elimina el elemento }\end{array}$ & $\begin{array}{l}\text { Correlación elemento- } \\
\text { total corregida }\end{array}$ & $\begin{array}{l}\text { Alfa de Cronbach si se } \\
\text { elimina el elemento }\end{array}$ \\
\hline VAR00001 & 126.6667 & 85.333 & .000 & .959 \\
\hline VAR00002 & 126.6667 & 85.333 & .000 & .959 \\
\hline VAR00003 & 127.0000 & 75.000 & 1.000 & .955 \\
\hline VAR00004 & 126.6667 & 85.333 & .000 & .959 \\
\hline VAR00005 & 127.0000 & 75.000 & 1.000 & .955 \\
\hline VAR00006 & 126.6667 & 85.333 & .000 & .959 \\
\hline VAR00007 & 126.6667 & 85.333 & .000 & .959 \\
\hline VAR00008 & 126.6667 & 85.333 & .000 & .959 \\
\hline VAR00009 & 127.0000 & 75.000 & 1.000 & .955 \\
\hline VAR00010 & 126.6667 & 85.333 & .000 & .959 \\
\hline VAR00011 & 126.6667 & 85.333 & .000 & .959 \\
\hline VAR00012 & 126.6667 & 85.333 & .000 & .959 \\
\hline VAR00013 & 127.0000 & 75.000 & 1.000 & .955 \\
\hline VAR00014 & 126.6667 & 85.333 & .000 & .959 \\
\hline VAR00015 & 126.6667 & 85.333 & .000 & .959 \\
\hline VAR00016 & 126.6667 & 85.333 & .000 & .959 \\
\hline VAR00017 & 127.0000 & 75.000 & 1.000 & .955 \\
\hline VAR00018 & 126.6667 & 85.333 & .000 & .959 \\
\hline VAR00019 & 126.6667 & 85.333 & .000 & .959 \\
\hline VAR00020 & 126.6667 & 85.333 & .000 & .959 \\
\hline VAR00021 & 126.6667 & 85.333 & .000 & .959 \\
\hline VAR00022 & 126.6667 & 85.333 & .000 & .959 \\
\hline VAR00023 & 127.0000 & 75.000 & 1.000 & .955 \\
\hline VAR00024 & 127.0000 & 75.000 & 1.000 & .955 \\
\hline VAR00025 & 127.0000 & 75.000 & 1.000 & .955 \\
\hline VAR00026 & 126.6667 & 85.333 & .000 & .959 \\
\hline
\end{tabular}


pp • 121-141 Juan Carlos Velásquez Díaz \& Lida Darley Pineda Rodríguez

\begin{tabular}{|c|c|c|c|c|}
\hline & $\begin{array}{l}\text { Media de la escala si } \\
\text { se elimina el elemento }\end{array}$ & $\begin{array}{l}\text { Varianza de la escala si } \\
\text { se elimina el elemento }\end{array}$ & $\begin{array}{l}\text { Correlación elemento- } \\
\text { total corregida }\end{array}$ & $\begin{array}{l}\text { Alfa de Cronbach si se } \\
\text { elimina el elemento }\end{array}$ \\
\hline VAR00027 & 126.6667 & 85.333 & .000 & .959 \\
\hline VAR00028 & 127.0000 & 75.000 & 1.000 & .955 \\
\hline VAR00029 & 127.0000 & 75.000 & 1.000 & .955 \\
\hline VAR00030 & 126.6667 & 85.333 & .000 & .959 \\
\hline VAR00031 & 126.6667 & 85.333 & .000 & .959 \\
\hline VAR00032 & 127.0000 & 75.000 & 1.000 & .955 \\
\hline VAR00033 & 127.0000 & 75.000 & 1.000 & .955 \\
\hline VAR00034 & 127.0000 & 75.000 & 1.000 & .955 \\
\hline VAR00035 & 126.6667 & 85.333 & .000 & .959 \\
\hline VAR00036 & 126.6667 & 85.333 & .000 & .959 \\
\hline VAR00037 & 126.6667 & 85.333 & .000 & .959 \\
\hline VAR00038 & 127.0000 & 75.000 & 1.000 & .955 \\
\hline VAR00039 & 126.6667 & 85.333 & .000 & .959 \\
\hline VAR00040 & 127.0000 & 75.000 & 1.000 & .955 \\
\hline VAR00041 & 126.6667 & 85.333 & .000 & .959 \\
\hline VAR00042 & 126.6667 & 85.333 & .000 & .959 \\
\hline VAR00043 & 127.0000 & 75.000 & 1.000 & .955 \\
\hline VAR00044 & 126.6667 & 85.333 & .000 & .959 \\
\hline VAR00045 & 126.6667 & 85.333 & .000 & .959 \\
\hline
\end{tabular}

\begin{tabular}{cc}
\hline Alfa de Cronbach & Número de Ítems \\
\hline .959 & 45 \\
\hline
\end{tabular}

\section{Análisis factorial exploratorio}

Se evaluaron estadísticamente los factores de la prueba (intimidador, víctima y observador) combinados con las dimensiones de la agresión (física, verbal y social), por lo que se sometieron a prueba 9 combinaciones en total, las cuales se describen a continuación:

Combinación 1. Factor Víctima Dimensión Agresión Física
Combinación 2. Factor Víctima Dimensión Agresión Social

Combinación 3. Factor Víctima Dimensión Agresión Verbal

Combinación 4. Factor Intimidador Dimensión Agresión Física

Combinación 5. Factor Intimidador Dimensión Agresión Social

Combinación 6. Factor Intimidador Dimensión Agresión Verbal 
Combinación 7. Factor Observador Dimensión Agresión Física

\section{Combinación 8. Factor Observador - Dimensión Agresión Social}

Combinación 9. Factor Observador Dimensión Agresión Verbal

En la Tabla 2 se pueden observar los resultados del análisis factorial exploratorio.

Los resultados identificaron que algunos ítems se encontraban dentro de dos factores distintos, es decir, que presentaron varianza superior a 1, razón por la que se depuró el instrumento, eliminando 10 ítems y consolidando la prueba en un total de 35 ítems, que son presentados en la Tabla 3.
Tabla 2.

Matriz factorial fase exploratoria.

\begin{tabular}{ccc}
\hline Ítems & Factor 1 & Factor 2 \\
\hline VAR00002 & .125 & .589 \\
VAR00003 & .092 & .239 \\
VAR00004 & .35 & .283 \\
VAR00006 & .346 & .109 \\
VAR00010 & .662 & .043 \\
VAR00012 & .238 & .186 \\
VAR00014 & .203 & .751 \\
VAR00015 & .029 & .116 \\
VAR00018 & .393 & .227 \\
VAR00019 & .289 & .632 \\
VAR00022 & .104 & .228 \\
VAR00024 & .576 & .125 \\
VAR00029 & .106 & .471 \\
VAR00030 & .561 & .323 \\
VAR00035 & .549 & -.196 \\
VAR00039 & .206 & .484 \\
VAR00040 & .599 & .064 \\
VAR00049 & .301 & .138 \\
\hline
\end{tabular}

Tabla 3.

Ítems definitivos del instrumento diseñado.

\begin{tabular}{|c|c|c|c|}
\hline No. & Ítems & Factor & $\begin{array}{l}\text { Dimensión } \\
\text { de agresión }\end{array}$ \\
\hline 1 & No permito que otros niños participen en actividades deportivas y recreativas & Intimidador & Social \\
\hline 2 & Algunos niños se alejan cuando quiero compartir con ellos & Víctima & Social \\
\hline 3 & He visto a niños rompiendo los objetos escolares de otros (cuadernos, libros, etc.) & Observador & Física \\
\hline 4 & Hablo mal de otros para dañar su buen nombre o reputación & Intimidador & Verbal \\
\hline 5 & $\begin{array}{l}\text { Envío mensajes hablando mal de otros niños por internet y } \\
\text { redes sociales (Facebook, Messenger, etc.) }\end{array}$ & Intimidador & Social \\
\hline 6 & Me ponen apodos o sobrenombres para molestarme & Víctima & Verbal \\
\hline 7 & He visto que agreden a otros niños con objetos (pistolas, cuchillos, palos, etc.) & Observador & Física \\
\hline 8 & Les pongo apodos o sobrenombres a otros niños para molestarlos & Intimidador & Verbal \\
\hline 9 & Ridiculizo a otros niños frente a los demás & Intimidador & Social \\
\hline 10 & He visto que a otros niños les roban sus objetos personales & Observador & Física \\
\hline 11 & Me lanzan objetos intencionalmente para lastimarme (piedras, balones, lápices, etc.) & Víctima & Física \\
\hline 12 & Se burlan de mí delante de los demás & Víctima & Verbal \\
\hline 13 & He visto que a otros niños les quitan las onces o el almuerzo & Observador & Física \\
\hline 14 & Trato mal a otros compañeros diciéndoles groserías & Intimidador & Verbal \\
\hline 15 & Me roban mis objetos personales & Víctima & Física \\
\hline 16 & Algunos niños me golpean (patadas, puños, empujones) & Víctima & Física \\
\hline 17 & $\begin{array}{l}\text { He visto que a otros niños les lanzan intencionalmente objetos } \\
\text { para lastimarlos (piedras, balones, lápices, etc.) }\end{array}$ & Observador & Física \\
\hline
\end{tabular}




\begin{tabular}{|c|c|c|c|}
\hline No. & Ítems & Factor & $\begin{array}{l}\text { Dimensión } \\
\text { de agresión }\end{array}$ \\
\hline 18 & A otros niños les ponen apodos o sobrenombres para molestarlos & Observador & Verbal \\
\hline 19 & He visto a compañeros que hablan mal de otros para dañar su buen nombre y reputación & Observador & Verbal \\
\hline 20 & Amenazo a otros con objetos (pistolas, cuchillos, palos, etc.) & Intimidador & Física \\
\hline 21 & $\begin{array}{l}\text { He visto que algunos compañeros ignoran a otros niños } \\
\text { intencionalmente para hacerlos sentir mal }\end{array}$ & Observador & Social \\
\hline 22 & Algunos niños me ignoran para hacerme sentir mal & Víctima & Social \\
\hline 23 & $\begin{array}{l}\text { He visto que compañeros hablan mal de otros por internet } \\
\text { y redes sociales (Facebook, Messenger, etc.) }\end{array}$ & Observador & Social \\
\hline 24 & He visto que culpan a otros niños de cosas que no han hecho para hacerlos quedar mal & Observador & Social \\
\hline 25 & Algunos niños les esconden objetos personales o escolares a otros para molestarlos & Observador & Social \\
\hline 26 & $\begin{array}{l}\text { Me envían mensajes con ofensas o insultos por Internet } \\
\text { y redes sociales (Facebook, Messenger, etc.) }\end{array}$ & Víctima & Social \\
\hline 27 & Les quito el dinero de las onces 0 almuerzo a otros niños & Intimidador & Física \\
\hline 28 & Me culpan de cosas que yo no hecho para hacerme quedar mal & Víctima & Social \\
\hline 29 & He visto a compañeros decir comentarios crueles para hacer sentir mal a otros & Observador & Verbal \\
\hline 30 & $\begin{array}{l}\text { He visto que se burlan de otros niños por sus características } \\
\text { físicas (color de piel, contextura física, etc.) }\end{array}$ & Observador & Verbal \\
\hline 31 & Algunos niños inventan chismes para dañar mi reputación & Víctima & Verbal \\
\hline 32 & Algunos niños me insultan y me dicen groserías & Víctima & Verbal \\
\hline 33 & He visto que a algunos niños no se les permite participar de juegos y actividades recreativas & Observador & Social \\
\hline 34 & Robo a otros niños sus objetos personales & Intimidador & Física \\
\hline 35 & Algunos niños me amenazan con lastimarme si no hago lo que quieren & Víctima & Verbal \\
\hline
\end{tabular}

\section{Análisis Factorial Confirmatorio}

El análisis factorial confirmatorio se realizó al igual que el exploratorio, por medio de la evaluación de las 9 combinaciones entre factores y dimensiones de la agresión; los resultados son presentados en la Tabla 4.

Tabla 4.

Resumen de Varianzas Totales

\begin{tabular}{|c|c|c|c|c|c|}
\hline Combinación Factores -Dimensiones & Factor 1 & Factor 2 & Factor 3 & Factor 4 & Factor 5 \\
\hline Víctima - Agresión Física & 1.453 & .985 & .562 & & \\
\hline Víctima - Agresión Social & 1.659 & .994 & .818 & .530 & \\
\hline Víctima - Agresión Verbal & 1.793 & .893 & .869 & .835 & .609 \\
\hline Intimidador - Agresión Física & 1.519 & .892 & .589 & & \\
\hline Intimidador - Agresión Social & 1.162 & .959 & .879 & & \\
\hline Intimidador - Agresión Verbal & 1.447 & .841 & .712 & & \\
\hline Observador - Agresión Física & 1.956 & .979 & .777 & .714 & .574 \\
\hline Observador - Agresión Social & 1.816 & .999 & .838 & .743 & .604 \\
\hline Observador - Agresión Verbal & 1.964 & .797 & .636 & .603 & \\
\hline
\end{tabular}

Nota. La Tabla 4 indica la unidimensional de cada una de las combinaciones. 
El cálculo del índice de confiabilidad instrumento la consistencia interna conse realizó por medio del Alfa de Cronbach, tinuaba siendo favorable. Los resultados con el objetivo de identificar si aún con se presentan en la Tabla 5.

la eliminación de ítems y depuración del

Tabla 5.

Coeficiente de Confiabilidad Alfa de Cronbach para la prueba final

\begin{tabular}{|c|c|c|c|c|}
\hline & $\begin{array}{c}\text { Media de la escala si } \\
\text { se elimina el elemento }\end{array}$ & $\begin{array}{l}\text { Varianza de la escala si } \\
\text { se elimina el elemento }\end{array}$ & $\begin{array}{l}\text { Correlación total } \\
\text { corregida }\end{array}$ & $\begin{array}{l}\text { A. Cronbach si se } \\
\text { elimina el elemento }\end{array}$ \\
\hline VAR00001 & 97.3333 & 65.333 & 1.000 & .957 \\
\hline VAR00002 & 97.0000 & 75.000 & .000 & .962 \\
\hline VAR00003 & 97.3333 & 65.333 & 1.000 & .957 \\
\hline VAR00004 & 97.0000 & 75.000 & .000 & .962 \\
\hline VAR00005 & 97.0000 & 75.000 & .000 & .962 \\
\hline VAR00006 & 97.0000 & 75.000 & .000 & .962 \\
\hline VAR00007 & 97.3333 & 65.333 & 1.000 & .957 \\
\hline VAR00008 & 97.0000 & 75.000 & .000 & .962 \\
\hline VAR00009 & 97.0000 & 75.000 & .000 & .962 \\
\hline VAR00010 & 97.3333 & 65.333 & 1.000 & .957 \\
\hline VAR00011 & 97.0000 & 75.000 & .000 & .962 \\
\hline VAR00012 & 97.0000 & 75.000 & .000 & .962 \\
\hline VAR00013 & 97.3333 & 65.333 & 1.000 & .957 \\
\hline VAR00014 & 97.0000 & 75.000 & .000 & .962 \\
\hline VAR00015 & 97.0000 & 75.000 & .000 & .962 \\
\hline VAR00016 & 97.0000 & 75.000 & .000 & .962 \\
\hline VAR00017 & 97.3333 & 65.333 & 1.000 & .957 \\
\hline VAR00018 & 97.3333 & 65.333 & 1.000 & .957 \\
\hline VAR00019 & 97.3333 & 65.333 & 1.000 & .957 \\
\hline VAR00020 & 97.0000 & 75.000 & .000 & .962 \\
\hline VAR00021 & 97.3333 & 65.333 & 1.000 & .957 \\
\hline VAR00022 & 97.0000 & 75.000 & .000 & .962 \\
\hline VAR00023 & 97.3333 & 65.333 & 1.000 & .957 \\
\hline VAR00024 & 97.3333 & 65.333 & 1.000 & .957 \\
\hline VAR00025 & 97.3333 & 65.333 & 1.000 & .957 \\
\hline VAR00026 & 97.0000 & 75.000 & .000 & .962 \\
\hline VAR00027 & 97.0000 & 75.000 & .000 & .962 \\
\hline VAR00028 & 97.0000 & 75.000 & .000 & .962 \\
\hline VAR00029 & 97.3333 & 65.333 & 1.000 & .957 \\
\hline VAR00030 & 97.3333 & 65.333 & 1.000 & .957 \\
\hline VAR00031 & 97.0000 & 75.000 & .000 & .962 \\
\hline VAR00032 & 97.0000 & 75.000 & .000 & .962 \\
\hline VAR00033 & 97.3333 & 65.333 & 1.000 & .957 \\
\hline VAR00034 & 97.0000 & 75.000 & .000 & .962 \\
\hline VAR00035 & 97.0000 & 75.000 & .000 & .962 \\
\hline
\end{tabular}

\begin{tabular}{cc}
\hline Alfa de Cronbach & Número de ítems \\
\hline .961 & 35
\end{tabular}




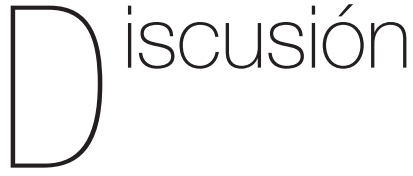

En la presente investigación se diseñó un instrumento para la identificación del matoneo, el cual incluye los conceptos básicos citados por Olweus (2006), quien define el matoneo como las acciones negativas que se producen de forma repetida en el tiempo, considerando que esta acción se produce cuando un estudiante, de manera intencionada, causa daño o hace sentir incómodo a alguno de sus pares, evidenciando en este acto un desequilibrio de fuerzas de relación de poder asimétrica.

Los análisis estadísticos de la prueba diseñada indican que ésta cumple adecuadamente con los criterios de confiabilidad y validez. Inicialmente se realizó la validación de contenido del instrumento por medio de la evaluación de jueces expertos, como lo describe Aroca (1999, citado por Delgado et al., 2002). Este es el método que más se utiliza para estimar este tipo de validez, esta evaluación permitió la depuración de los ítems en cuanto a su redacción, relevancia y pertinencia, por lo que se modificaron parcialmente los ítems 4, 21, 25, 30, 39, 40, $41 y 42$.

Para establecer el criterio de confiabilidad se utilizó el Coeficiente Alfa de Cronbach. Una vez analizados los resultados se logró establecer que la prueba evaluada por los jueces expertos obtuvo adecuados índices que Confiabilidad, de acuerdo con Kerlinger y Lee (2002) se observa que los índices de confiabilidad de un instrumento no deben ser inferiores a .8, afirmando así que existe un nivel satisfactorio de confiabilidad. Los valores del Alfa de Cronbach oscilan entre 0 y 1 , donde los valores cercanos a 1 (superiores a .8) indican que el instrumento es fiable y que tiene mediciones estables y consistentes, mientras que los resultados inferiores a .8 son indicadores de que el instrumento de evaluación presenta una variabilidad heterogénea en los ítems o preguntas que lo componen, por lo que sus resultados pueden ser equívocos. Para el desarrollo del instrumento actual se puede decir que los resultados del Alfa de Cronbach de .959 en la prueba inicial indican que existe una adecuada consistencia interna y que el instrumento tiene adecuados índices de confiabilidad.

Una vez modificados los ítems recomendados por los jueces expertos y hallado el coeficiente de confiabilidad de la prueba se aplicó el instrumento de evaluación a la muestra, con el objetivo de realizar la validación de constructo, utilizando el método del análisis factorial. Argibay (2006) indica que esta forma de evaluar la validez se utiliza cuando el test está dividido en factores, debido a que desde la teoría del instrumento se plantean los distintos factores como atributos diferenciados, mencionando además que el número de sujetos no debe ser menor a cinco veces la cantidad de ítems del instrumento, razón por la que se aplicó la prueba a 300 estudiantes, cumpliendo de esta forma con los requerimientos necesarios para la realización del análisis factorial.

Como se observa en los resultados, el análisis factorial se realizó con la combinación de los factores de la prueba (intimidador, víctima y observador) y las 
diferentes dimensiones de la agresión (física, social y verbal), logrando abarcar de esta forma los principales aspectos teóricos del matoneo.

El análisis factorial exploratorio mostró que algunos ítems no discriminaban adecuadamente, por lo que se indicó dentro de dos factores distintos, es decir, que se presentó varianza superior a 1 en cada uno de ellos. Una vez obtenidos estos indicadores se revisaron nuevamente los ítems diseñados inicialmente, con el objetivo de depurar cada uno de los factores, consolidando de esta forma la prueba final para ser sometida al análisis factorial confirmatorio. Los ítems eliminados fueron: 1, 2, 11, 15, 19, 22, 27, 29,31 y 39.

Dentro del análisis estadístico también se estableció la medida de adecuación muestral propuesta por de Kaiser, Meyer, y Olkin (KMO), que es un índice que toma valores entre 0 y 1 , se utiliza para comparar las magnitudes de los coeficientes de correlación observados con las de correlación parcial, señalando de esta forma si es deseable el realizar un análisis factorial, donde puntajes iguales o superiores a .5 suponen que es aceptable el planteamiento de realizar un análisis factorial, puntajes cercanos a la unidad señalan buenos índices para desarrollar el proceso en mención. Para las 9 combinaciones de factores y dimensiones se obtuvieron puntuaciones superiores al indicador mínimo propuesto por de Kaiser, Meyer, y Olkin. En orden consecutivo, de acuerdo a las combinaciones, los resultados encontrados fueron: 1. Combinación, .506; 2. Combinación, .585; 3. Combinación, .668; 4. Combinación, .540; 5. Combinación, .525; 6. Combinación, .583; 7.
Combinación, .698; 8. Combinación, .0652; 9. Combinación, .715. Además, se estableció el indicador de la prueba de esfericidad de Bartlett, el cual permite identificar si existen relaciones significativas entre las variables, al encontrarse puntajes inferiores a .005. Para todas las combinaciones evaluadas se encontraron puntajes positivos de $.000 \mathrm{a}$ excepción de la combinación Factor Intimidador-Dimensión Agresión Social, en la que se encontró un puntaje negativo de .105, indicando relación no significativa entre las variables a evaluar.

Uno de los indicadores de mayor relevancia que se obtuvo en el análisis estadístico de la prueba dentro del análisis factorial confirmatorio es el que se refiere a la varianza total por combinación de factores y dimensiones. Este indicador es favorable cuando existe un solo factor con valores propios iguales o superiores a 1, indicando que es factor unidimensional y que los ítems analizados se encuentran dentro del mismo factor de evaluación. Para todas las combinaciones analizadas de factores y dimensiones se encontraron puntajes positivos, es decir, que en cada una de ellas los ítems evaluados se agrupan apropiadamente dentro del mismo factor unidimensional.

Una vez realizado el análisis factorial confirmatorio se estableció el Coeficiente de Confiabilidad Alfa de Cronbach para los resultados de la prueba aplicada a la muestra, de acuerdo a la combinación de todos los factores y dimensiones, midiendo la consistencia del instrumento aplicado, donde se establece que existe una adecuada consistencia interna y que el instrumento tiene adecuados índices de confiabilidad al obtener un puntaje de .847 . 
Con la prueba para la identificación del matoneo ya definitiva se obtuvo nuevamente el índice de confiabilidad de la misma por medio del Alfa de Cronbach, con el objetivo de identificar si aun con la eliminación de ítems y depuración del instrumento los índices de consistencia interna continuaban siendo favorables. Así, los resultados del Alfa de Cronbach de .961 indican que existe una adecuada consistencia interna y que el instrumento tiene adecuados índices de confiabilidad.

Los datos descritos en los resultados de la investigación muestran que el instrumento diseñado cumple con los dos criterios psicométricos con los que debe contar todo instrumento de evaluación, logrando obtener adecuados índices en referencia a confiabilidad y validez. Algunos otros estudios como el de Varela, Farren, y Tijimes (2010) han evaluado estadísticamente pruebas de convivencia escolar por medio de los criterios de confiabilidad y validez, utilizando también el método referido al Análisis Factorial y al coeficiente Alfa de Cronbach, evaluando de igual manera factores que involucran los tres actores presentes dentro del matoneo y distintas clases de violencia, aunque ésta incluye aspectos adicionales como el porte ilegal de armas, el consumo de sustancias psicoactivas y el maltrato ejercido por adultos. Así, como en la presente investigación, la desarrollada por estos autores logró establecer en sus escalas adecuados índices de confiabilidad y discriminación.

Como conclusión de la presente investigación se puede decir que se logró cumplir con el objetivo general de la misma, en lo que se refiere a diseñar un instrumento de evaluación psicológica que permita identificar matoneo dentro de contextos educativos y clínicos, para lo cual se utilizaron estrategias psicométricas para el establecimiento de los criterios de validez (de contenido y constructo) y confiabilidad. Como sugerencia para generar continuidad en la investigación se recomienda iniciar su proceso de estandarización en una muestra significativa para la población colombiana.

Aunque no era uno de los objetivos de la investigación se realizó un breve análisis de las pruebas aplicadas, encontrando que de la muestra seleccionada de 300 estudiantes solo 2 reportan ser víctimas del matoneo, lo que corresponde a un .66\% del total de niños y niñas evaluadas. No se encontró ningún estudiante con el perfil de intimidador, posiblemente porque estos no reconocen en algunos casos las consecuencias de sus actos o no los observan como negativos.

En lo que se refiere a observadores se encontraron 13 estudiantes que indican presenciar comportamientos de matoneo, lo que corresponde a un $4.33 \%$ del total de la muestra. Estos datos se muestran como consistentes de acuerdo a los reportes brindados por los funcionarios de la institución educativa, quienes indican que los casos reportados por posibles conductas de matoneo son mínimos. En lo que respecta a los observadores identificados no se encuentra reporte por parte de estos a funcionarios del colegio, utores como Serrano et al. (2005) describen que, por lo general, la mayoría de ellos no denuncian este tipo de comportamientos. 


\section{Referencias}

Aiken, L. (1996). Tests psicológicos de evaluación. México, D.F.: Prentice-Hall.

Arbigay, J. (2006). Técnicas psicométricas, Cuestiones de Validez y Confiabilidad. Revista Subjetividad y Procesos Cognitivos, 8, 15-33.

Arellano, N. (2008). Violencia entre pares escolares (bullying) y su abordaje a través de la mediación escolar y los sistemas de convivencia. Revista Informe de Investigaciones Educativas, 2, 211 - 230.

Arroyave, P. (2012). Factores de vulnerabilidad y riesgo asociados al bullying. Revista CES Psicología, 5(1), 116-125.

Bonilla, E., Osorio, J., \& Triana, S (2010). Diseño de un instrumento para evaluar el "bullying" agresor y la víctima desde el agresor. (Tesis de grado). Universidad Católica de Colombia, Bogotá.

Cerezo, F. (2002). La Violencia en las Aulas. Análisis y propuestas de intervención. Madrid, España: Pirámide.

Cerezo, F. (2006). Violencia y Victimización entre escolares. El Bullying: Estrategias de identificación y elementos para la intervención a través del Test Bull-S. Revista Electrónica de Investigación Psicoeducativa, 9(2), 1-20. Recuperado el 15 de Junio de 2013 de http://www. investigacionpsicopedagogica.org/ revista/articulos/9/espannol/Art_9_115. pdf
Chaux, E. (2008). Manejo de casos de intimidación escolar: El Método de Preocupación Compartida. Colombia: Pontificia Universidad Javeriana.

Chaux, E. (2009). La chispita que quería encender todos los fósforos: Percepciones, creencias y emociones frente a la intimidación en un colegio masculino. Voces y Silencios: Revista Latinoamericana de Educación, 1(1), 5-22.

Chaves, C., Morales., \& Ortega. (2015). Tipo de agresión resultado de un problema y conflicto en la población adolescente de siete instituciones públicas de educación media de San Juan de Pasto. Revista Ciencia y Cuidad 12(1), 76-92.

Cohen, R., \& Swerdlink, M. (2001). Pruebas y evaluación psicológicas. Introducción a las pruebas y a la medición. México, D.F.: McGraw Hill.

Cronbach, L. (1951). Coefficient alpha and the internal structure of tests. Revista psychometrika, 16(3), 297-334

Delgado, Y., Colombo, L., \& Rosmel, O. (2002). Conduciendo la Investigación. Caracas: Comala.

Erazo, O. (2012). La intimidación escolar, actores y características. Revista Vanguardia Psicológica 3(1), 80-102.

Garaigordobil, M. (2011). Prevalencia y consecuencias del cyberbullying: una revisión. Revista International Journal of Psychology and Psychological Therapy, 11(2), 233-254. 
Hernández, R., Fernández, C., \& Baptista, P. (2006). Metodología de la investigación. México, D.F: McGraw Hill.

Jaramillo, P. (2012). A prueba de Bullies. Investigación internacional sobre el acoso escolar. Revista comunicación y relaciones públicas. Recuperado el 20 de agosto de 2013 de http://repositorio.usfa. edu.ec/handle/23000/1722.

Juárez, F., \& Montejo, M. (2006). Propiedades psicométricas del inventario de situaciones y comportamientos agresivos y del inventario de motivos para la agresión. Revista Universitas Psychological, 7, 149-171.

Kerlinger, F., \& Lee, H. (2002). Investigación del Comportamiento. Métodos de Investigación en Ciencias Sociales. México: D.F.: McGraw Hill.

Kumpulainen, K. (2008). Psychiatric conditions associated with bullying; International Journal Adolesc. Medical and Health, 20(2), 121-32.

Luna, I. (2006). El Acoso escolar no es un juego de niños. Asociación Colombiana de Psiquiatría. Recuperado el 27 de julio de 2012 de www.psiquiatria.org.co/ .../B/ boletín...6/boletin_electronico_6.asp.

Mera, L. (2014). Caracterización y efectos psicosomáticos del matoneo escolar en la institución educativa de los Uvos, del municipio de Popayán. Recuperado el 10 de Febrero de 2016 de www. http://repository.unad.edu.co/ handle/10596/2773

Ministerio de Educación Nacional. (2013, Marzo). Ley 1620 de 2013, sistema nacional de convivencia escolar y formación para el ejercicio de los derechos humanos, la educación para la sexualidad y la prevención y mitigación de la violencia escolar". Recuperado en Junio 11, 2013 de http://wsp. presidencia.gov.co/Normativa/Leyes/ Documents/2013/LEY\%201620\%20 DEL\%2015\%20DE\%20MARZO\%20 DE\%202013.pdf.

Moreno, M., Vacas, C., \& Roa, J. (2006). Victimización escolar y clima socio familiar. Revista Iberoamericana de educación. No. 40/6. Recuperado el 17 de Julio de 2013 de http://www.rieoei. org/investigacion/1608Moreno.pdf

Olweus, D. (1999). The nature of school bullying: A cross-national perspective. EEUU: Catalano.

Olweus, D. (2006). Conductas de acoso y amenaza entre escolares. Madrid, España: Morata S.L. Tercera Edición.

Ricou, J. (2005). Acoso escolar. Sevilla, España: Rd Editores.

Rodríguez, R., Seoane., A., \& Pedreira, J. (2008). Niños Contra Niños: El Bullying Como Trastorno Emergente. Revista Anales de Pediatría, 64(2), 162-186.

Rodríguez, A. (2012). Consecuencias del matoneo escolar. La importancia de creer que el mundo es justo. (Trabajo de Grado). Universidad del Rosario, Bogotá. Recuperado el 05 de Febrero de 2016 de http://www. http:// repository.urosario.edu.co/bitstream/ handle/10336/4069/1020731168-2012. pdf?sequence $=1$.

Serrano, A., \& Iborra, I (2005). Violencia entre compañeros en la escuela. Valencia: 
Centro Reina Sofía para el Estudio de la Violencia.

Tornimbeni, S., Pérez, E., Olaz, F., \& Fernández, A. (2004). Introducción a los Tests Psicológicos. ( $3^{\mathrm{a}}$ Ed.). Córdoba, Argentina: Ed. Brujas

Varela, J., Farren, D., \& Tijimes, C. (2010). Validación de un instrumento para medir la violencia escolar. Fundación Paz Ciudadana. Chile. Recuperado el 20 de Mayo de 2013 de http://es.scribd.com/ doc/46415133/Validacion-Instrumentomedicion-violencia-escolar.
Velásquez, J., Pineda, L., Castro, C. (2013). Diseño de un instrumento para la identificación del matoneo. (Tesis de grado). Universidad de la Sabana: Bogotá. Recuperado el día 20 de junio de 2016 de intellectum.unisabana.edu. co/handle/10818/9065.

Voors, W. (2005). Bullying el acoso escolar. Barcelona: Oniro. 